C2000 IEEE. Personal use of this material is permitted. However, permission to reprint/republish this material for advertising or promotional purposes or for creating new collective works for resale or redistribution to servers or lists, or to reuse any copyrighted component of this work in other works must be obtained from the IEEE. 


\title{
Selective Broadcast during Asynchronous Pre-meeting Stage of Strategy Formulation: A GSS capability for Indonesian Culture
}

\author{
Sjarif Abdat, Douglas J. Atkinson and Graham P. Pervan \\ School of Information Systems - Curtin University of Technology \\ \{AbdatS, AtkinsonD, PervanG\}@cbs.curtin.edu.au
}

\begin{abstract}
Research about Group Support Systems and the design of these technologies uses western culture as a point of reference. While the research reveals promising results on the effectiveness of these technologies, the technologies may be less effective when applied directly to different cultures or cross-cultural situations where there may be different support requirements. This paper presents the concept of Selective Broadcast which was originally defined as the delivery of a packet to a specified subset of possible destinations [22], but in this paper refers to the distribution of information to specified members of a communication group. It is potentially one of the most significant requirements of technological support for groups engaged in the premeeting stage of strategy formulation in Indonesia. Indonesian culture has relatively high power distance, low individualism, and weak uncertainty avoidance [12, 13], and high context [10, 11]. Selective Broadcast is discussed with respect to meetings, group memory, culture and practical applications.
\end{abstract}

\section{Introduction}

In the era of widespread use of collaborative technology, including team and small group activities, significant work is done in meetings. Most of the research and development of collaborative technology has been conducted by North American organizations and universities using North American subjects. Basing this technology on the needs of western culture may lead to resistance and ineffectiveness when it is used in other national cultures.

GSS meeting designers need to be culturally sensitive, they have to match the tools and communication configuration of a GSS while at the same time meeting goals and cultural norms [23]. Based on observation of Singaporean groups they suggest that, "harmony might be enhanced by using a GSS to create a distributed meeting in which group members are physically separated and information exchange is asynchronous. In this case members can carefully asses others opinions and weigh their thoughts before making a statement" (p.53).

A recent assessment of 200 different controlled experiments on GSS research found that most of the experiments have been conducted in same-time/sameplace, and there have been no published experiments using 'anytime/anyplace' [8]. Another recent literature analysis from 234 GSS publications between 1984 and 1996, which included not only controlled experiments research but also field and case studies, found that $82.5 \%$ research papers referred to face-to-face situations [17]. This is also the case when national culture was an issue, as most GSS research was focused on how GSS has an effect during face-to-face meetings. There is a need to examine the relationship between national culture and GSS for the group activities outside the face-to-face meeting, namely during the 'pre-meeting' and 'postmeeting' stages.

GSS capabilities can be analyzed along four dimensions: process support, process structure, task support and task structure [15]. In a study on the use of a GSS in the strategic planning process of thirty organizations, the ability of the GSS to provide process support was found to be the most important contributor to strategic planning success [7]. Process support has positive effects not only on production and identification of information, but also on communication and integration of information. In general, task structure and process structure are found to be secondary contributors while task support contributed little. Process support has to do with the communication infrastructure within which members of the group interact, including parallelism, anonymity and group memory [15].

Abdat and Pervan [1] suggest that parallelism might not be such a significant GSS capability in asynchronous group activities since the interactions are at different times. Watson, Ho and Raman [23] found that anonymity might be inappropriate in certain circumstances for Singaporeans and suggested that this feature should be switchable. De Vreede [21] points to a negative aspect of the group memory GSS capability when he argues that:

"With respect to group memory, the other side of the coin is that, people may hesitate to contribute if everything is stored immediately. It may be the case that the more sensitive brainstorming and decision making process are impaired if all of the output is documented" (p.129).

He suggests that people's position on certain topics may 
either become more rigid or less outspoken for fear of not having the possibility of changing one's point of view later on in the process [21].

All of the above findings and argumentation on GSS process support capabilities suggest the need for communication flexibility as the settings change. To support this flexibility the concept of selective broadcast $[9,22]$ is introduced, which is borrowed from computer network terminology and was originally defined as "the delivery of a packet to a specified subset of possible destinations" [22]. In this paper it is defined as "the distribution of information to specified members of a communication group" [1].

This paper discusses why selective broadcast becomes one of the most significant GSS requirements in the Indonesian culture at the pre-meeting stage of strategy formulation. In section 2 , we discuss cultural issues and how these influence typical Indonesian meetings. This is followed in section 3 by an explanation of the GSS requirements for an asynchronous pre-meeting. In section 4 the selective broadcast concept is presented in the paper. Section 5 contains some discussion on implementation of selective broadcast and section 6 contains conclusions and a discussion of plans for future development and empirical work in this area.

\section{Typical Indonesian Meetings}

Hofstede [12, 13] defines culture as "the collective programming of the mind which distinguishes the members of one group of people from those of another". There are some key studies about national culture dimensions that are believed to be stable over time. The culture dimensions that will be used in this research are power distance, individualism, uncertainty avoidance and context.

Power distance is the extent to which the less powerful members of institutions and organizations within a country expect and accept that power is distributed unequally [13]. In low power distance countries, status differences among individuals are less significant, and people believe in the legitimate use of power and having equal rights. In high power distance countries, status differences among individuals are very pronounced.

Individualism pertains to societies in which the ties between individuals are loose: everyone is expected to look after himself or herself and his or her immediate family. Collectivism as its opposite pertains to societies in which people from birth onwards are integrated into strong, cohesive in-groups, which throughout a person's lifetime continue to protect them in exchange for unquestioning loyalty [13].

Uncertainty avoidance can be defined as the extent to which the member of a culture feels threatened by uncertain or unknown situations. This feeling is, among other things, expressed through nervous stress and in a need for predictability: a need for written and unwritten rules [13]. Weak uncertainty avoidance countries are characterized by people who are comfortable in ambiguous situations and with unfamiliar risks.

Context is the information that surrounds an event; it is inextricably bound up with the meaning of that event $[10,11]$. The elements that combine to produce a given meaning, event and context are in different proportions depending on the culture. The cultures of the world can be compared on a scale from high to low context. A high context communication is one in which most of the information is already in the person, while very little is in the coded, explicit, transmitted part of the message. A low context communication is just the opposite; the mass of the information is vested in the explicit code.

In the Indonesian culture, which has low individualism, high context, and high power distance, people prefer to arrive at major meetings with all details negotiated so that there are no surprises, which may lead to "loss of face". The term "loss of face" relating to protecting your "face", is actually one of Confucian work dynamism [4]. This reflects the 'high context' attitude of Indonesians. These face-to-face meetings are usually attended by a large number of people, including many only loosely related to the task, which reflects the 'high collectivism' attitude of Indonesians where there is a conscious desire to get everyone involved (and is also related to high context). The result is usually already known because most of the work has been done before the meeting. In such a culture and resulting process, Indonesians tend to pay more attention to the premeeting of the meeting cycle.

In theory one might believe that a national culture with strong uncertainty avoidance might seek a premeeting to overcome the uncertainty before coming to the 'in-meeting'. However, analysis by Hofstede reveals that Indonesia is one of the weak uncertainty avoidance countries. This finding would seem to indicate that at least as far as the need of pre-meeting is concerned, other dimensions of cultures such as power distance, individualism and context might have stronger influence.

Abdat and Pervan [1] proposed a model using a basic meeting cycle to show how events surrounding the meeting are interrelated. This model also can be used to reveal how different cultural dimension affect the different stages of the meeting cycle (see figure 1). Definitions for the events are as follows:

1. Meeting: a group information process to achieve specific goals.

2. In-meeting: a scheduled event in which all participants of the meeting have a possibility to undertake communication and interaction at the same time (synchronous).

3. Pre-meetings: unscheduled events in which subsets of the participants of the meeting are able to communicate and interact, either at the same time (synchronous) or different time (asynchronous) before the 'in-meeting'. The purpose of these activities may be to exchange information, discuss ideas, negotiate, and seek consensus before coming 
to the 'in-meeting'.

4. Post-meetings: unscheduled events in which subsets of the participants of the meeting have a possibility to communicate and interact, either at the same time (synchronous) or different time (asynchronous) after the in-meeting. The purpose of these activities may be to clarify details from the 'in-meeting', and to organise and carry out the implementation of decisions made.

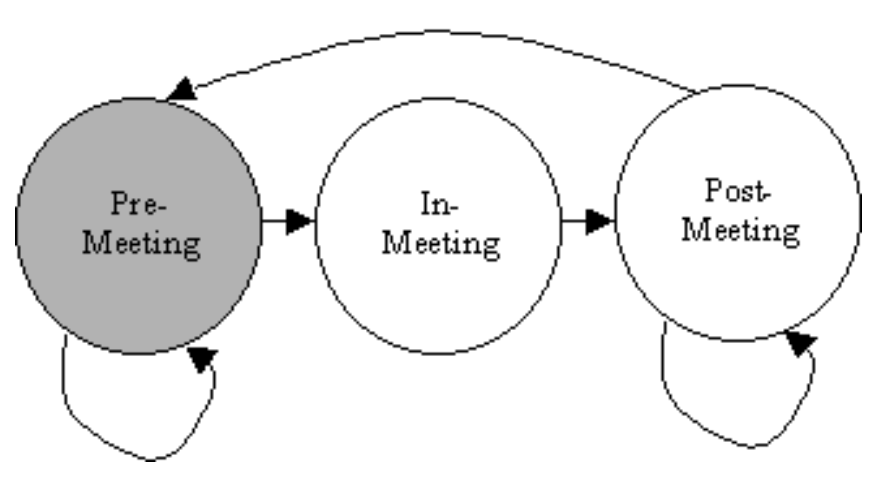

Figure 1. Basic Meeting Cycle

At the pre-meeting and post-meeting stages the communication and interaction are not only between the participants of the 'in-meeting' but may involve communication with other persons (see an example on Figure 2). In the discussions that follow, the "group" is synonymous with the "in-meeting participants".

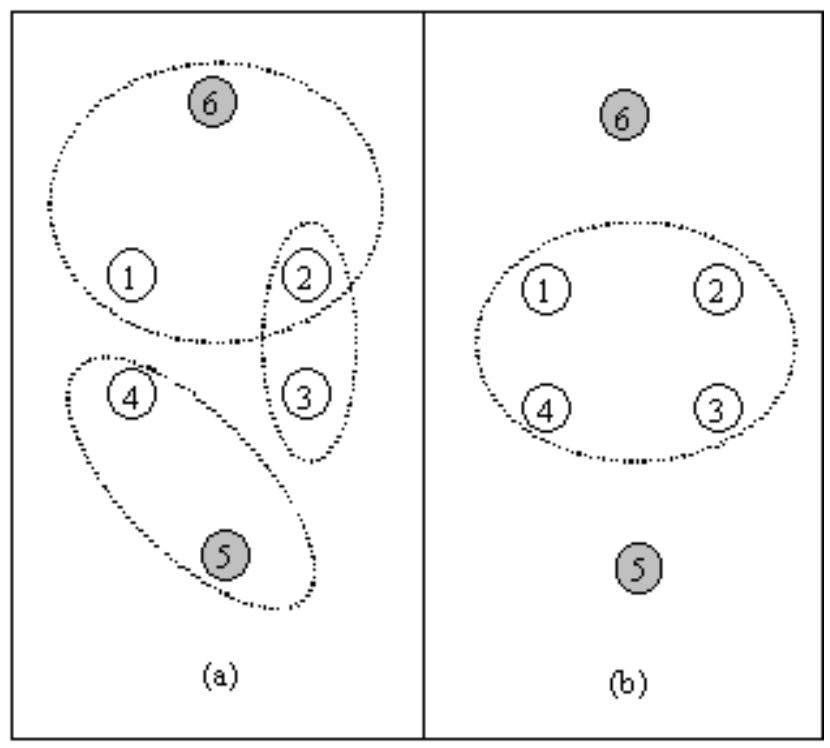

Figure 2. Pre-Meeting and In-Meeting Communication

Figure 2 shows an example of a communication group consisting of four 'in-meeting' participants $\{1,2,3,4\}$ and two from outside of the 'in-meeting' participants $\{5,6\}$. During the pre-meetings shown in figure 2(a), there are three communication sub-groups and all of them are subsets of the communication group. In figure 2(c), the communication is only among the 'in-meeting' participants.

Several authors $[3,16,18,19]$ have discussed why the pre-meeting is important. The pre-meeting provides participants with sufficient context and perspective about the tasks to be accomplished. Therefore participants are coming to meetings better prepared [6]. This is very relevant to the high context cultures. Moreover there are relationships according to Hofstede, in which collectivism (low individualism) tends to be high context.

The following sections of the paper will focus only on Asynchronous Pre-meetings.

\section{GSS Requirements for Asynchronous Pre-meetings}

GSS requirements for asynchronous communication in general have been proposed and discussed by Turoff [20]. The GSS requirements described in this section are not even intended to replace the GSS requirements during face to face meetings. They should be viewed as a complementary and synergistic to the face-to-face requirements. There are additional requirements for premeetings and we can reuse of some previously defined capabilities by taking into account different time requirements and cultural appropriateness.

There are technological systems that can be used to enhance work in groups. They can be classified into four major types, on the basis of function(s) that they serve for the group: inter-member communication, extra-group communication, information/data access, and member task interaction [14]. Moreover, the type of information/data should not be restricted to the specific media such as text only, but also include multimedia as the technology available to handle it.

General requirements, based on the definition of an asynchronous pre-meeting, are related to communication and memory aspects, as follows:

- Internal Group Communication: meeting participants should be able to communicate with all participants or subsets of the participants at any time.

- External Group Communication: meeting participants should be able to communicate with other relevant individuals or groups outside of the meeting participants at any time.

- Group Memory: meeting participants should be able to access information or knowledge created by the group and made available for the group.

- Public Memory: meeting participants should be able to access information or knowledge from sources outside the group, and selected individuals outside the group should be able to access information or knowledge made available to outsiders by the group.

- Sub-Group Memory: meeting participants should be able to access information or knowledge which is created by a subset of the participants or selected external individuals outside of the participants of the 
meeting and made available only for specific individuals.

In considering culture-based requirements to be taken into account for Indonesians, high power distance effects can (theoretically) be reduced using anonymity. However, Watson, Ho and Raman [23] found that this feature might be inappropriate in certain circumstances for Singaporeans. This may also be the case in the Indonesian culture, so it is suggested that the use of anonymity should be switchable. It could then be used in specific situations, such as when there is a mix of high and low status participants, and the group wishes to reduce the status differential.

As indicated by Dennis et al. [7], process support is a key element of success in GSS support of strategy formulation in the face-to-face setting. Abdat and Pervan, [1] suggest that this is also likely to be the case in a dispersed and asynchronous setting. The key process support capabilities are summarised in Table 1 below, along with the corresponding needs in a dispersed, asynchronous setting which have been identified so far, and the Indonesian cultural aspects of these support needs.

\begin{tabular}{|l|l|}
\hline $\begin{array}{l}\text { Process Support Capabilities } \\
\text { (Asynchronous Pre-meeting) }\end{array}$ & Related Cultural Issues \\
\hline Selective broadcast & Power Distance, Context \\
\hline Anonymity & Power Distance \\
\hline Memory & Context, Individualism \\
\hline
\end{tabular}

Table 1. The key process support capabilities for asynchronous pre-meetings

\section{The Concept of Selective Broadcast during Asynchronous Pre-meetings}

Selective broadcast is defined as "the capability of group support systems to distribute information to specified members of a communication group". While, in face-to-face meetings, information is broadcast to all participants, in the pre-meeting stage a subset of the participants have a possibility of communicating among themselves and may involve people outside the meeting participants. McGrath and Hollingshead [14] introduced implicitly the capability of selective broadcast when they defined the capability of GCSS (Group Communication Support Systems) and GXSS (Group External Communication Support Systems).

One might think that the selective broadcast capability just utilizes email functions. While email does have a selective broadcast capability, in itself it is not sufficient to handle the impact of the selective broadcast needs such as shared documents. Moreover email as a system lacks the process support and structure to guide the group interactions required of an asynchronous GSS. The combination of email capability and information sharing can more effectively utilize memory capability through the tracking, organizing and storage of all sub- group communication in a database.

In certain situations selective broadcast can be used to overcome the negative impacts of high power distance culture during asynchronous pre-meetings and so obviate the need for anonymity. For example, when an individual wants to initiate a new idea but does not want to be noticed by the whole group until the ideas have matured enough to be fully shared and recorded.

As indicated by de Vreede [21], people may hesitate to contribute if everything is stored immediately. During asynchronous pre-meetings the use of selective broadcast can become a medium to achieve a certain stage of 'content-maturity' before becoming group memory. In the Indonesian culture, which has the cultural characteristic of low individualism, individuals tend to think carefully about whether or not the idea or opinion will be useful for others. Therefore, instead of broadcasting the idea or opinion, individuals usually check and verify their points with a colleague or superior. In this situation selective broadcast may have a positive effect with group memory by segmenting the memory process capability into group, sub-group and private memory.

At the early stage of strategy formulation, activities such as idea generation using electronic brainstorming can be held during the asynchronous pre-meetings. There are some possible impacts of introducing selective broadcast during asynchronous pre-meeting to the group process regardless of the cultural dimensions, such as to avoid information overloading, filter group memory and function as incubator for a premature idea or opinion.

In Indonesian culture the impact of the use of selective broadcast might be even more pronounced for specific cultural dimension such as high power distance, low individualism and high context. Examples may include:

(a) Criticism from another contributor may be received without 'loss of face' in front of the whole group.

(b) The effect of power distance may be accommodated here by allowing participants to consult their superiors first before broadcasting their ideas/opinions, and they may be able to let their ideas/opinions be used by the superior or another member who has more influence in the group.

(c) Consensus might be even easier to achieve since the initiator and contributors are personally involved and working together in developing an idea.

\section{Implementation of Selective Broadcast}

The implementation of selective broadcast is part of the development of RAPAT (Replicable Asynchronous Premeeting Application Template), an asynchronous GSS for the Indonesian culture at the pre-meeting stage of strategy formulation [1,2]. After exploration of the initial requirements for asynchronous pre-meetings and evaluation of the available tools and development environments, Lotus Notes ${ }^{\circledR}$ (currently known as Domino $^{\circledR}$ ) was chosen as the development environment 


\section{for RAPAT.}

Currently, Domino ${ }^{\circledR}$ is the dominant commercial groupware product. Features of Domino ${ }^{\circledR}$ include email, calendaring, discussion databases, document-databases, workflow and web integration. Application development can be undertaken making use of templates, and a scripting language, LotusScript. Enhancements are possible using HTML, JavaScript and Java applets. Comprehensive security is provided via authentication, database access control lists, field protection and encryption. Domino ${ }^{\circledR}$ operates in a client server environment and works on all of the common networks and operating systems. Support for mobile computing allows end users to work off-line and synchronize or replicate databases with servers when required. It is thus particularly suited to support participants who collaborate at different times and in different places during asynchronous pre-meetings.

Selective broadcast could be partially implemented in Domino $^{\circledR}$ making use of the "views" feature. Views enable a summary selection of documents. The selection can be programmed based on fields and formulae. For example, a selected view of incoming and outgoing communications could include one-to-one and one-tomany correspondences. The ordering by communication partners could be extended to documents received as email attachments. In theory this could be extended to digital voice communication.

Based on TeamRoom [5], a Domino ${ }^{\circledR}$ template, the system is being developed and will be used to study the pre-meeting stage of strategy formulation for some case studies in Indonesian organizations. While Domino ${ }^{\circledR}$ has a capability to define all the users belonging to specified groups or roles on its public address book under central administration control, each user can define a private address book to include other members under his/her control. This function can then be used and refined under the TeamRoom template. TeamRoom has a field to send information to a group and/or a specified sub-group, and each participant can specify to whom the information should be automatically forwarded by defining conditions. For example, if the subject is related to the "quality of service" then correspondence would be forwarded to all customer service officers.

TeamRoom has a capability to link each communication during pre-meeting to the specified event. This event might be one of the 'in-meeting' stages or other related deadlines. In addition, the group has to specify the type and the category of the communication. In this situation participants can categorize task-related information such as SWOT (Strength, Weakness, Opportunity and Threat) analysis, and can add the context of the strategy formulation task by including mission statements relevant to the specific situation. Although Domino ${ }^{\circledR}$ and the TeamRoom template seem to fulfil most of the requirements, some parts need modification to be more culturally sensitive to the Indonesian context.
Currently RAPAT is being used and reviewed by a group of Indonesian researchers in Western Australia to develop a strategic plan for their organization. The following example is captured from one of the use-cases, namely "Initiate Idea/Issue" (see figure 3). The user is provided with the facilities to view available information by category, communication type and person. From this view the user can compose a new idea by clicking "New Idea/Issue" button. The user has access to other facilities from this view, including a searching facility and managing display using Previous-Next and Expand-

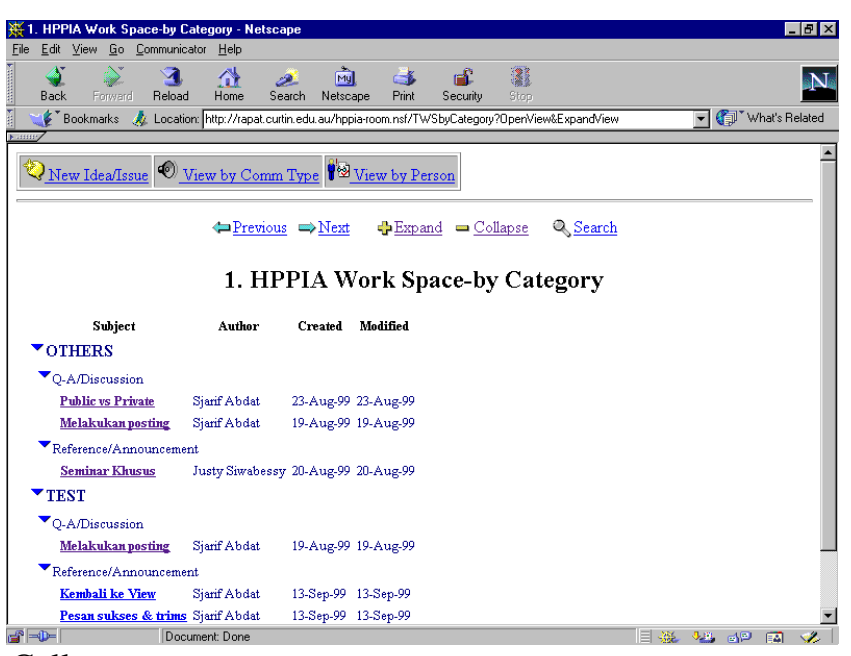

Collapse.

Figure-3, View available information

The following screen (Figure 4), shows how the user can compose a message for raising an issue, which is one of the communication types. This message by default will be sent to all participants (Broadcast), but in this example the user decides to send the message to specific members of the group by selecting their names (Selective Broadcast).

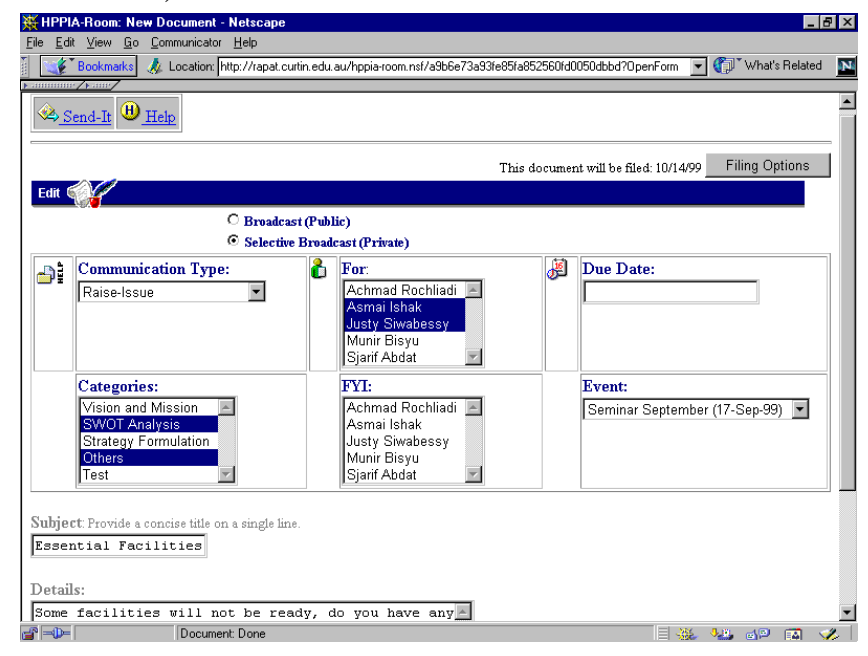

Figure-4, Example of RAPAT screen to initiate idea/issue

A message can be viewed from one or more categories. By choosing more than one category, and a recipient can find the message listed in different views. Events can be 
included to view a message grouping by the specific event. After the user has finished filling in the subject and details of the message, the user can send the message by clicking on the Send-It button on the top of the page.

\section{Conclusions and Future work}

In this paper we have proposed selective broadcast as a GSS capability to be used during the asynchronous premeeting stage of strategy formulation in Indonesian culture. The discussion indicates that some process support capabilities expected in the face-to-face setting such as anonymity and memory are also needed in the dispersed, asynchronous setting, and cultural dimensions such as power distance, context, and individualism may affect the design of these support capabilities. Asynchronous pre-meeting requires selective broadcast as one addition to the GSS capabilities for process support. In addition, selective broadcast might be used to avoid the use of anonymity and to enhance the group memory.

It is intended that selective broadcast will be embedded in some typical group support technologies and tested in case studies in Indonesia. Through this process the empirical results will be used to review the design, specific tools and interfaces to customize for the specific needs of this particular non-western culture.

\section{References}

[1] S. Abdat and G. P. Pervan, "Developing Group Support Systems for Indonesian Culture at the Pre-Meeting Stage of Strategy Formulation: A Proposal and Some initial Requirements", Proceeding of the 5th International Conference of the International Society for Decision Support Systems, Melbourne, July 20-23, 1999.

[2] S. Abdat and G. P. Pervan, "Development of RAPAT: Asynchronous GSS at the Pre-meeting Stages of Strategy Formulation for Indonesian Culture", Proceeding of the 10th Australasian Conference of Information Systems, Wellington, New Zealand, December 1-3, 1999.

[3] R. Bostrom and R. Anson, "The face-to-face Electronic Meeting: A Tutorial," in Computer Augmented Teamwork: A Guided Tour, R. Bostrom, R. Watson, and S. Kinney, Eds. New York: Van Nostrand Reinhold, 1992, pp. 16-33.

[4] CCC, "Chinese Values and the Search for Culture-Free Dimensions of Culture," Journal of Cross-Cultural Psychology, vol. 18, 1987, pp. 143-164.

[5] P. Cole and E. C. Johnson, "Lotus Development: Team Room - A Collaboration Workspace for Cross Functional Teams," in Transforming Organisations Through Groupware: Lotus Notes in Action, P. Lloyd and R. Whitehead, Eds. London: Springer-Verlag, 1996, pp. 2339.

[6] E. Darr, "Australian Bureau of Statistics: Universal Adoption," in Transforming Organisations Through Groupware: Lotus Notes in action, P. Lloyd and R. Whitehead, Eds. London ; New York: Springer-Verlag, 1996, pp. 70-79.

[7] A. R. Dennis, C. K. Tyran, D. R. Vogel, and J. F. Nunamaker, Jr., "Group Support Systems for Strategic Planning," Journal of Management Information Systems, vol. 14, 1997, pp. 155-184.

[8] J. Fjermestad and S. R. Hiltz, "An Assessment of Group Support Systems Experimental Research: Methodology and Results," Journal of Management Information Systems, vol. 15, 1999, pp. 7-149.

[9] A. J. Frank, L. D. Wittie, and A. J. Bernstein, "Multicast Communication on Network Computer," IEEE Software, vol. 2, 1985, pp. 49-61.

[10] E. T. Hall, Beyond culture. Garden City, New York.: Anchor Press, 1976.

[11] E. T. Hall and M. H. Hall, Understanding Cultural Differences. Yarmouth, Maine: Intercultural Press, Inc., 1990.

[12] G. H. Hofstede, Culture's consequences, international differences in work-related values. Beverly Hills, Calif.: Sage Publications, 1980.

[13] G. H. Hofstede, Cultures and organizations: software of the mind. London; New York: McGraw-Hill, 1991.

[14] J. E. McGrath and A. B. Hollinghead, "Putting the "Group" Back in Group Support Systems: Some Theoretical Issues About Dynamic Processes in Groups with Technological Enhancements.," in Group Support Systems: New Perspectives, L. M. Jessup and J. S. Valacich, Eds. New York: Macmilian Publishing Company, 1993, pp. 78-96.

[15] J. F. Nunamaker, A. R. Dennis, J. S. Valacich, D. R. Vogel, and J. F. George, "Electronic Meeting Systems to Support Group Work," Communications of the ACM, vol. 34, 1991, pp. 40-61.

[16] L. Oppenheim, "Making Meetings Matter: A Report to the 3M Corporation," 3M Meeting Management Institute, Austin, TX 1987.

[17] G. P. Pervan, "A review of research in Group Support Systems: Leaders, approaches and directions," Decision Support Systems, vol. 23, 1998, pp. 149-159.

[18] H. B. Schwartzman, The Meeting: Gatherings in Organizations and Communities. New York: Plenum Press, 1989.

[19] R. M. Schwarz, The Skilled Facilitator: Practical Wisdom for Developing Effective Groups, 1st ed. San Francisco: Jossey-Bass Publishers, 1994.

[20] M. Turoff, "Computer-Mediated Communication Requirements for Group Support," Journal of Organizational Computing, 1991, pp. 85-113.

[21] G. J. de Vreede, "Facilitating Organizational Change: The Participative Application of Dynamic Modelling," Doctoral dissertation in School of Systems Engineering, Policy Analysis and Management. Delft: Delft University of Technology, Netherland, 1995, pp. 335.

[22] D. W. Wall, "Selective Broadcast in Packet-Switched Networks", Proceeding of the Sixth Berkeley Workshop Distributed Data Management and Computer Network, Berkeley, February, 1982.

[23] R. Watson, T. Ho, and K. Raman, "Culture - A Fourth Dimension of Group Support Systems," Communications of the ACM, 1994, pp. 44-55. 